\title{
Multi-choice stochastic transportation problem involving Weibull distribution
}

\author{
Deshabrata Roy Mahapatra \\ Department of Mathematics, Kishorenagar Sachindra Siksha Sadan \\ Contai, East Medinipore-721401, West Bengal, India \\ Email: dmdesh123@gmail.com
}

(Received July 16, 2013; in final form December 13, 2013)

\begin{abstract}
This paper explains the important role in application of stochastic distribution and multichoice framework on the field of transportation environment. The purpose of this paper is to provide a solution procedure to multi-choice stochastic transportation problem involving the parameters as supply and demand of Weibull distribution and cost coefficients of a single criterion of minimization of objective function which are multi-choice in nature. At first, all stochastic constraints are transformed into deterministic constraints by using the stochastic approach. Recently, Mahapatra et al. [14] have proposed a methodology to transfer the multi-choice stochastic transportation problem to an equivalent mathematical programming model which can accumulate a maximum of eight choices on the cost coefficients of the objective function. In this paper, a generalized transformation technique is also present to discuss the two types of transformation technique. Using any one of the transformation technique, the decision maker can handle a parameter of the cost coefficients of objective function with finite number of choice associated with additional restriction for obtaining the equivalent deterministic form. Finally, a numerical example is provided to validate the theoretical development and solution procedure.
\end{abstract}

Keywords: Multi-choice programming; stochastic programming; weibull distribution; transportation problem; transformation technique; mixed-integer programming

AMS Classification: 90C08; 90C15; 990C11

\section{Introduction}

A transportation problem refers to a class of linear programming problems that involves selection of most economical shipping routes for transfer of a uniform commodity from a number of sources to a number of destinations. A stochastic transportation model in which the constraints are stochastic in nature and the cost coefficients are multi choice type is considered. These two contributions are mainly responsible for the development of this transportation models. The above models deals with transportation of a product available at several sources to a number of different destinations. Each shipping sources has a certain capacity and each destination has a certain requirement associated with a certain cost of shipping from sources to the destinations. The objective is to minimize the cost of transportation while meeting the requirements at the destinations. Transportation problems may also involve movement of product from plants to warehouses, warehouses to warehouses, warehouses to retailers and retailers to customers.

In transportation problem, there are in $m$ sources and $n$ destinations. Let $C_{i j}^{k}$ of the $k$-th objective function could represent the unit of transportation cost for transporting the unit from $i$-th $(i=1,2, \cdots, m)$ origin to $j$-th $(j=1,2, \cdots, n)$ destination, $x_{i j}$ is the quantity shipped from the $i$-th origin to $j$ - th destination, $a_{i}$ is the supply available at origin $i$ and $b_{j}$ is the destination $j$ In such a situation, all these parameters of all 
constraints are defined as random variables to follow Weibull distribution. Thus there exists multi-choice penalty criteria in the cost coefficients of transportation which leads to the research work on multi-choice stochastic transportation problem. Most of real world problems of practical importance are modeled nowadays with multi-choice parameters. Hitchcock [11] first presented a study entitled " The Distribution of a Product from Several Sources to Numerous Localities". The presentation is regarded as the first important contribution to the solution of transportation problems. Koopmans [12] presented a study called "Optimum Utilization of the Transportation System". These two contributions are mainly responsible for the development of transportation models which involve a number of shipping sources and a number of destinations.

In real-world application, it is very difficult to know all the information about the input parameters of the mathematical programming model because relevant data are in existent or scarce, difficult to obtain or to estimate, the system is subject to changes, and so forth, that is, input parameters are uncertain. One of the best ways of modeling these uncertainties in the form of mathematical programming is known as stochastic programming. It is widely used in several research areas such as agriculture, capacity planning, finance, forestry, military, production control and scheduling, telecommunication, transportation, environmental management planning etc. Goicoechea et al. [10] discussed the deterministic equivalents for some probabilistic programming involving normal and other distributions. Mahapatra et al. [13], have presented the solution procedure of multi-objective stochastic transportation problem incorporating normal distribution with joint constraints. The mathematical formula of the Weibull distribution is defined as follows: a random variable $x$ is said to be Weibull distributed if its probability density function is given by

$$
\begin{array}{r}
f(x ; \alpha, \beta)=\frac{\alpha}{\beta^{\alpha}} x^{\alpha-1} e^{\left\{-\left(\frac{x}{\alpha}\right)^{\alpha}\right\}} \\
x \geq 0 \text { and } \alpha>0, \beta>0,
\end{array}
$$

where $\alpha$ and $\beta$ are the shape and scale parameters. The Weibull distribution has been found to be useful for describing cost times and lifetimes in reliability applications. It is distribution that received maximum attention in the past few decades. Weibull distribution may be a good choice to describe the data on lifetimes or strength data, in some practical situations it fits worse than its competitions. In this manner, it is applied in risk analysis, actuarial science and engineering. Furthermore, the Weibull distribution has applications in medical, biological, transportation planning and earth sciences etc. Weibull Wallodi [17], a Sweden physicist in 1950's, first introduced the Weibull distribution, it has become a useful data treatment method in reliability studies of materials and electronic devices. The Weibull distribution is practically and theoretically applicable to the studies of estimation of the future probability under a certain loading by Fok [9].

Multi-choice programming is a mathematical programming problem, in which the decision maker is allowed to set multiple number of choices for a parameter. In recent years, methods of multi-choice stochastic optimization become increasingly important in solving scientifically decision making problems arising in economics, industry, health care, transportation, agriculture, military, engineering and technology. Biswal and Acharya [2] proposed transformation techniques to transform multi-choice linear programming problem to an equivalent mathematical model in which constraints are associated with multi-choice parameters. In 2007, Chang [4], [5] presented multi-choice goal programming model with limited number of choices for a parameter with multiplicative terms of binary variables using continuous variable. In other paper Chang [6], he has also proposed a revised method for multi-choice goal programming model which does not involve multiplicative terms of binary variables to model the multiple aspiration levels. Chang et al. [7] discussed the present situation of commutative business environment. Hence to find ways maximize profit and to minimize total costs, group pricing discrimination strategy has become an important issue for decision makers. Biswal and Acharya [3] discussed the solving for probabilistic programming problem involving multi-choice parameters. Acharya et al. [1] presented a generalized form of new transformation technique of multi-choice linear programming problem. Liao [8] have proposed an alternative method for solving the multi-segment goal programming problems with two contributions: (1) the alternative method represents a linear from of multi-segment goal programming which can easily be solved by common linear programming sties, and (2) the alternative approach does not involve multiplicative terms of binary variables, this leads to more efficient use of multisegment goal programming and is easily understood by industrial participants. Ravindran et 
al. [15] have presented a mathematical model in which an appropriate constraint is to be chosen using binary variables. The present paper considers both randomness and multi-choice simultaneously in the application area of transportation planning frame work. Two different types of stochastic inequality constraints are associated with the problem where the random variables follow Weibull distribution. The cost coefficients of the objective function are multi-choice type, where a generalized transformation technique is established for transforming the multichoice transportation problem to an equivalent mathematical programming model. Using any one of these transformation techniques, the transformed model can be derived. Binary variable and additional restrictions are introduced to formulate a non-linear mixed integer programming model. In order to solve the present problem, a new methodology has been proposed to solve multi-choice stochastic transportation problem. The remainder of the paper is organized as follows: following the introduction in Section 1, mathematical model of multi-choice stochastic transportation problem involving Weibull distribution is presented in Section 2. The generalized transformation techniques to derive equivalent mathematical models of multi-choice stochastic transportation problem involving Weibull distribution are presented in Section 3. In order to verify the proposed transformation techniques, a case study is presented in Section 4. Finally, conclusions and points towards directions for future research are presented in Section 5 respectively.

\section{Mathematical Model}

In this paper, a mathematical model for multichoice stochastic transportation problem involving Weibull distribution is considered as follows:

\section{Model 1}

$$
\min : z=\sum_{i=1}^{m} \sum_{j=1}^{n}\left\{C_{i j}^{1}, C_{i j}^{2}, \cdots, C_{i j}^{k}\right\} x_{i j},
$$$$
k=1,2, \cdots, K
$$

subject to $\operatorname{Pr}\left(\sum_{j=1}^{n} x_{i j} \leq s_{i}\right) \geq 1-\gamma_{i}$,

$$
\begin{array}{r}
i=1,2, \cdots, m \\
\operatorname{Pr}\left(\sum_{i=1}^{m} x_{i j} \geq d_{j}\right) \geq 1-\delta_{j}, \\
j=1,2, \cdots, n \\
x_{i j} \geq 0, \quad \forall i \text { and } j
\end{array}
$$

where $0<\gamma_{i}<1, \forall i$ and $0<\delta_{j}<1, \forall j$. It is assumed that $s_{i} \quad(i=1,2, \cdots, m)$ and $d_{j} \quad(j=1,2, \cdots, n)$ are Weibull random variables and $C_{i j}^{k}=\left\{C_{i j}^{1}, C_{i j}^{2}, \cdots, C_{i j}^{k}\right\}$ is a multichoice parameter.

The following cases are to be considered

1. Only $s_{i},(i=1,2, \cdots, m)$ follows Weibull distribution.

2. Only $d_{j},(j=1,2, \cdots, n)$ follows Weibull distribution.

3. Both $s_{i},(i=1,2, \cdots, m)$ and $d_{j},(j=$ $1,2, \cdots, n)$ follow Weibull distribution.

\subsection{Only $s_{i},(i=1,2, \cdots, m)$ follows Weibull distribution}

It is assumed that $s_{1}, s_{2}, \ldots, s_{m}$ are independent random variables and $s_{i},(i=1,2, \ldots, m)$ follows Weibull distribution with shape and scale parameters as $\alpha_{i}$ and $\beta_{i}$ respectively, where the aspiration level is $\gamma_{i}, \quad 0<\gamma_{i}<1$. The constraint (3) can be rewrite as below:

$\operatorname{Pr}\left(\sum_{j=1}^{n} x_{i j} \leq s_{i}\right) \geq 1-\gamma_{i}, \quad i=1,2, \cdots, m$.

The probability density function of $s_{i}(i=$ $1,2, \cdots, m)$ is given by

$$
\begin{array}{r}
f\left(s_{i} ; \alpha_{i}, \beta_{i}\right)=\frac{\alpha_{i}}{\beta_{i}^{\alpha_{i}}} s_{i}^{\alpha_{i}-1} e^{\left\{-\left(\frac{s_{i}}{\alpha_{i}}\right)^{\alpha_{i}}\right\}} \\
s_{i} \geq 0 \text { and } \alpha_{i}>0, \beta_{i}>0 .
\end{array}
$$

Hence the probabilistic constraint can be presented as:

$$
\int_{\sum_{j=1}^{n} x_{i j}}^{\infty} f\left(s_{i} ; \alpha_{i}, \beta_{i}\right) d\left(s_{i}\right) \geq 1-\gamma_{i} .
$$

The above integral can be expressed as:

$$
\int_{\sum_{j=1}^{n} x_{i j}}^{\infty} \frac{\alpha_{i}}{\beta_{i}^{\alpha_{i}}} s_{i}^{\alpha_{i}-1} e^{\left\{-\left(\frac{s_{i}}{\alpha_{i}}\right)^{\alpha_{i}}\right\}} d\left(s_{i}\right) \geq 1-\gamma_{i} .
$$

Let, $\left(\frac{s_{i}}{\beta_{i}}\right)^{\alpha_{i}}=z$. The above constraints (8) can be rewrite as:

$$
\int_{\left(\frac{\sum_{j=1}^{n} x_{i j}}{\beta_{i}}\right)^{\alpha}{ }_{i}}^{\infty} e^{-z} d(z) \geq 1-\gamma_{i}
$$

which can be integrated as:

$$
-\left[e^{-z}\right]_{\left(\frac{\sum_{j=1}^{n} x_{i j}}{\beta_{i}}\right)^{\alpha_{i}}}^{\infty} \geq 1-\gamma_{i} .
$$

It can be further simplified as:

$$
e^{-\left(\frac{\sum_{j=1}^{n} x_{i j}}{\beta_{i}}\right)^{\alpha_{i}}} \geq 1-\gamma_{i} .
$$

Taking logarithm in both sides twice,

$$
\alpha_{i} \ln \left(\frac{\sum_{j=1}^{n} x_{i j}}{\beta_{i}}\right) \leq \ln \left\{-\ln \left(1-\gamma_{i}\right)\right\} .
$$


After simplifying and rearranging the above constraints cab be stated as:

$$
\ln \left(\sum_{j=1}^{n} x_{i j}\right) \leq \ln \beta_{i}+\frac{1}{\alpha_{i}} \ln \left\{-\ln \left(1-\gamma_{i}\right)\right\} .
$$

Thus finally, the probabilistic constraints (3) can be transformed into a deterministic linear constraints as:

$$
\sum_{j=1}^{n} x_{i j} \leq e^{\left[\ln \beta_{i}+\frac{1}{\alpha_{i}} \ln \left\{-\ln \left(1-\gamma_{i}\right)\right\}\right]} .
$$

Thus, a multi-choice deterministic transportation problem (see Model 2) instead of multi-choice stochastic transportation model can be stated as follows.

\section{Model 2:}

$$
\begin{gathered}
\min : z=\sum_{i=1}^{m} \sum_{j=1}^{n}\left\{C_{i j}^{1}, C_{i j}^{2}, \cdots, C_{i j}^{k}\right\} x_{i j}, \\
k=1,2, \cdots, K \\
\text { subject to } \quad \sum_{j=1}^{n} x_{i j} \leq e^{\left[\ln \beta_{i}+\frac{1}{\alpha_{i}} \ln \left\{-\ln \left(1-\gamma_{i}\right)\right\}\right]} \\
\sum_{i=1}^{m} x_{i j} \geq d_{j}, j=1,2, \cdots, n \\
\text { where, } \sum_{i=1}^{m} e^{\left[\ln \beta_{i}+\frac{1}{\alpha_{i}} \ln \left\{-\ln \left(1-\gamma_{i}\right)\right\}\right]} \geq \sum_{j=1}^{n} d_{j}
\end{gathered}
$$

(feasibility condition).

\subsection{Only $d_{j},(j=1,2, \cdots, n)$ follows Weibull distribution}

It is assumed that $d_{1}, d_{2}, \ldots, d_{n}$ are independent random variables and $d_{j}(j=1,2, \ldots n)$ follows Weibull distribution with shape and scale parameters as $\alpha_{j}^{\prime}$ and $\beta_{j}^{\prime}$ respectively, where the aspiration levels is $\delta_{j}, 0<\delta_{j}<1$. The constraint of Model 1 can be written as follows:

$\operatorname{Pr}\left(\sum_{i=1}^{m} x_{i j} \geq d_{j}\right) \geq 1-\delta_{j}, \quad j=1,2, \cdots, n$.

Rearranging the above constraints can be written as,

$$
\operatorname{Pr}\left(\sum_{i=1}^{m} x_{i j} \leq d_{j}\right) \leq \delta_{j}, j=1,2, \cdots, n .
$$

The probability density function of $d_{j} \quad(j=$ $1,2, \cdots, n)$ is given by

$$
\begin{aligned}
& f\left(d_{j} ; \alpha_{j}^{\prime}, \beta_{j}^{\prime}\right)=\frac{\alpha_{j}^{\prime}}{\beta_{j}^{\prime \alpha_{j}^{\prime}}} d_{j}^{\alpha_{j}^{\prime}-1} e^{\left\{-\left(\frac{d_{j}}{\alpha_{j}^{\prime}}\right)^{\alpha_{j}^{\prime}}\right\}} \\
& d_{j} \geq 0 \text { and } \alpha_{j}^{\prime}>0, \quad \beta_{j}^{\prime}>0 .
\end{aligned}
$$

Hence the probabilistic constraint can be presented as:

$$
\int_{\sum_{i=1}^{m} x_{i j}}^{\infty} f\left(d_{j} ; \alpha_{j}^{\prime}, \beta_{j}^{\prime}\right) d\left(d_{j}\right) \leq \delta_{j} .
$$

The above integral can be expressed as:

$$
\int_{\sum_{i=1}^{m} x_{i j}}^{\infty} \frac{\alpha_{j}^{\prime}}{\beta_{j}^{\prime \alpha_{j}^{\prime}}} d_{j}^{\alpha_{j}^{\prime}-1} e^{\left\{-\left(\frac{d_{j}}{\alpha_{j}^{\prime}}\right)^{\alpha_{j}^{\prime}}\right\}} d\left(d_{j}\right) \leq \delta_{j}
$$

Let, $\left(\frac{d_{j}}{\beta_{j}^{\prime}}\right)^{\alpha_{j}^{\prime}}=z^{\prime}$ The above integral $(22)$ can be rewrite as:

$$
\int_{\left(\frac{\sum_{i=1}^{m} x_{i j}}{\beta_{j}^{\prime}}\right)_{j}^{\alpha \prime}}^{\infty} e^{-z^{\prime}} d\left(z^{\prime}\right) \leq \delta_{j},
$$

which can be integrated as:

$$
-\left[e^{-z^{\prime}}\right]_{\left(\frac{\sum_{i=1}^{m} x_{i j}}{\beta_{j}^{\prime}}\right)^{\alpha_{j}^{\prime}}}^{\infty} \leq \delta_{j}
$$

It can be further simplified as:

$$
e^{-\left(\frac{\sum_{i=1}^{m} x_{i j}}{\beta_{j}^{\prime}}\right)^{\alpha \prime}} \leq \delta_{j} .
$$

Taking logarithm in both sides twice,

$$
\alpha_{j}^{\prime} \ln \left(\frac{\sum_{i=1}^{m} x_{i j}}{\beta_{j}^{\prime}}\right) \geq \ln \left\{-\ln \left(\delta_{j}\right)\right\} .
$$

After rearranging of the above constraint can be written as:

$$
\ln \left(\sum_{i=1}^{m} x_{i j}\right) \geq \ln \beta_{j}^{\prime}+\frac{1}{\alpha_{j}^{\prime}} \ln \left\{-\ln \left(\delta_{j}\right)\right\} .
$$

Thus finally, the probabilistic constraints (4) can be transformed into a deterministic linear constraints as,

$$
\sum_{i=1}^{m} x_{i j} \geq e^{\left[\ln \beta_{j}^{\prime}+\frac{1}{\alpha_{j}^{\prime}} \ln \left\{-\ln \left(\delta_{j}\right)\right\}\right]}
$$

Thus, a multi-choice deterministic transportation problem (see Model 3) instead of multichoice stochastic transportation problem can be expressed as follow. 


\section{Model 3:}

$$
\begin{array}{r}
\min : z=\sum_{i=1}^{m} \sum_{j=1}^{n}\left\{C_{i j}^{1}, C_{i j}^{2}, \cdots, C_{i j}^{k}\right\} x_{i j}, \\
\text { subject to } \quad \sum_{j=1}^{n} x_{i j} \leq s_{i}, \quad i=1,2, \cdots, K \\
\sum_{i=1}^{m} x_{i j} \geq e^{\left[\ln \beta_{j}^{\prime}+\frac{1}{\alpha_{j}^{\prime}} \ln \left\{-\ln \left(\delta_{j}\right)\right\}\right]} \\
\text { where, } \sum_{i=1}^{m} s_{i} \geq \sum_{j=1}^{n} e^{\left[\ln \beta_{j}^{\prime}+\frac{1}{\alpha_{j}^{\prime}} \ln \left\{-\ln \left(\delta_{j}\right)\right\}\right]},
\end{array}
$$

(feasibility condition).

\subsection{Both $s_{i},(i=1,2, \cdots, m)$ and $d_{j},(j=1,2, \cdots, n)$ follow Weibull distributions}

The shape and scale parameters of the random variables $s_{i}$ and $d_{j}$ are known and previously defined. In this case, both $s_{i}$ and $d_{j}$ follow Weibull distribution. Model $\mathbf{1}$ is transferred into an equivalent deterministic model as:

\section{Model 4:}

$$
\begin{aligned}
\min : z & =\sum_{i=1}^{m} \sum_{j=1}^{n}\left\{C_{i j}^{1}, C_{i j}^{2}, \cdots, C_{i j}^{k}\right\} x_{i j}, \\
k=1,2, \cdots, K & \\
\text { subject to } & \sum_{j=1}^{n} x_{i j} \leq e^{\left[\ln \beta_{i}+\frac{1}{\alpha_{i}} \ln \left\{-\ln \left(1-\gamma_{i}\right)\right\}\right]} \\
& \left.\sum_{i=1}^{m} x_{i j} \geq e^{\left[\ln \beta_{j^{\prime}}{ }^{\prime}+\frac{1}{\alpha_{j}}\right.} \ln \left\{-\ln \left(\delta_{j}\right)\right\}\right]
\end{aligned}
$$

where

$$
\begin{array}{r}
\sum_{i=1}^{m} e^{\left[\ln \beta_{i}+\frac{1}{\alpha_{i}} \ln \left\{-\ln \left(1-\gamma_{i}\right)\right\}\right]} \geq \\
\sum_{j=1}^{n} e^{\left[\ln \beta_{j^{\prime}}+\frac{1}{\alpha_{j^{\prime}}} \ln \left\{-\ln \left(\delta_{j}\right)\right\}\right]}
\end{array}
$$

(feasibility condition).

\section{Generalized Transformation of the Objective Function}

\subsection{Transformation technique: 1}

The proposed model is derived for restriction on the upper bound of binary variables on cost coefficients of the objective function:

\section{Case 1:}

$$
\begin{array}{r}
\min : z=\sum_{i=1}^{m} \sum_{j=1}^{n}\left\{C_{i j}^{1}, C_{i j}^{2}, \cdots, C_{i j}^{n_{s}}\right\} x_{i j}, \\
s=1,2, \cdots, K
\end{array}
$$

The total number of choices for first step is $k_{s}$. We proposed that $k_{s} \geq 2$.

Case 2: The different models also depend upon the set of conjugate terms of binomials coefficients of whose sum is closer or nearer to the aspiration levels or choices.

a. In every different models, the restriction of $\left(2^{n s}-k_{s}\right)$ indicates the total number of constraints in which firstly there have been auxiliary constraints and in rest of them (constraints) in restriction there also additional constraints.

b. The similar models are also performed in each different models which can depend upon the same restriction, associated with others additional constraints.

Case 3: The number of binary variables for each aspiration levels is required to handle the multi-choice parameters in cost coefficients of the objective function in the following manner.

Find $n_{s}$, for which $2^{\left(n_{s}-1\right)}<k_{s}<2^{n_{s}}$, where $n_{s}$ are represented as the number of binary variables. Let binary variables are $z_{i j}^{1}, z_{i j}^{2}, \cdots, z_{i j}^{n_{s}}$; where $k_{s}$ the number of choices.

Case 4: Expand $2^{n_{s}}={ }^{n_{s}} \quad C_{0}+{ }^{n_{s}} C_{1}+{ }^{n_{s}}$ $C_{2}+\cdots+{ }^{n_{s}} C_{r_{s 1}-1}+{ }^{n_{s}} C_{r_{s 1}}+{ }^{n_{s}} C_{r_{s 1}+1}+{ }^{n_{s}}$ $C_{r_{s 1}+2}+\cdots+{ }^{n_{s}} C_{r_{s 2}-1}+{ }^{n_{s}} C_{r_{s 2}}+{ }^{n_{s}} C_{r_{s 2}+1}+{ }^{n_{s}}$ $C_{r_{s 2}+2} \cdots+{ }^{n_{s}} C_{n_{s}-1}+{ }^{n_{s}} C_{n_{s}}$ and select the smallest number of consecutive terms whose sum is "equal to" or " just greater than" $k_{s}$ from the expression. Let the terms ${ }^{n_{s}} C_{r_{s 1}},{ }^{n_{s}} C_{r_{s 1}+1},{ }^{n_{s}} C_{r_{s 1}+2}, \cdots,{ }^{n_{s}} C_{r_{s 2}-1},{ }^{n_{s}} C_{r_{s 2}}$ are associated to respect the lower and upper bounds.

Case 5: When $2^{n s}=k_{s}$, the choices of $k_{s}$ is equal to the expansion of $2^{n s}$. Then, there does not appear to the introduction of auxiliary and additional constraints involving the binary variables.

Case 6: Assigned $k_{s}$ binary codes to $k_{s}$ number of aspiration levels for cost coefficients of the objective function. 


$$
\begin{aligned}
& \min : z=\left[\sum_{i=1}^{{ }^{n_{s}} C_{r_{s 1}}} \sum_{j=1}^{{ }^{n_{s}} C_{r_{s 1}}} A_{i j}^{r_{s 1}} B_{i j}^{r_{s 1}} C_{i j}^{k}+\right. \\
& \sum_{i=1}^{n_{s}} \sum_{j=1}^{C_{r_{s 1}+1}{ }^{n_{s}}} \sum_{i j}^{C_{r_{s 1}+1}} A_{i j}^{r_{s 1}+1} B_{i j}^{r_{s 1}+1} C_{i j}^{\left\{n_{s} C_{r_{s 1}}+k\right\}}+\text {, } \\
& \cdots,+\sum_{i=1}^{{ }^{n_{s}} C_{r_{s 2}-1}} \sum_{j=1}^{n_{s}} A_{r_{s 2}-1} A_{i j}^{r_{s 2}-1} B_{i j}^{r_{s 2}-1} \\
& C_{i j}^{\left[\left\{{ }^{n_{s}} C_{r_{s 1}}+, \cdots,+{ }^{n_{s}} C_{r_{s 2}-2}\right\}+k\right]} \\
& \left.+\sum_{i=1}^{\left(k_{s}-L_{i j}^{1}\right)} \sum_{j=1}^{\left(k_{s}-L_{i j}^{1}\right)} A_{i j}^{r_{s 2}} B_{i j}^{r_{s 2}} C_{i j}^{\left(L_{i j}^{1}\right)+k}\right] x_{i j}, \\
& s=1,2, \cdots, K
\end{aligned}
$$

where, $L_{i j}^{(1)}={ }^{n_{s}} C_{r_{s 1}}+{ }^{n_{s}} C_{r_{s 1}+1}+{ }^{n_{s}} C_{r_{s 1}+2}+$ $\cdots+{ }^{n_{s}} C_{r_{s 2}-1}$;

$j_{1}^{\prime} \in\left\{1,2, \cdots,\left(n_{s}-s\right)+1\right\}, j_{2}^{\prime} \in\left\{2,3, \cdots,\left(n_{s}-\right.\right.$ $s)+2\}, \cdots, j_{s}^{\prime} \in\left\{s, s+1, \cdots, n_{s}\right\}$;

$I_{s}^{\left(j^{\prime}\right)}=\left\{\left(j_{1}^{\prime}, j_{2}^{\prime}, \cdots, j_{s}^{\prime}\right) / j_{1}^{\prime}<j_{2}^{\prime}<\cdots<j_{s}^{\prime}, s=\right.$ $\left.r_{s 1}, r_{s 1+1}, r_{s 1+2}, \cdots, r_{s 2}\right\}$

$A_{i j}^{s_{k}}=\left\{z_{i j}^{\left(j_{1}^{\prime}\right)}, z_{i j}^{\left(j_{2}^{\prime}\right)}, \cdots, z_{i j}^{\left(j_{s}^{\prime}\right)} /\left(j_{1}^{\prime}, j_{2}^{\prime}, \cdots, j_{s}^{\prime}\right)\right.$

$\left.\in I_{s}^{\left(j^{\prime}\right)}\right\}$;

$s=r_{s 1}, r_{s 1}+1, r_{s 1}+2, \cdots, r_{s 2}$.

Therefore, $B_{i j}^{s_{k}}=\left\{\Pi_{k=1}^{n_{s}}\left(1-z_{i j}^{j_{k}}\right) / j_{k}^{\prime}\right.$ not belongs to $\left.\left(j_{1}^{\prime}, j_{2}^{\prime}, \cdots, j_{s}^{\prime}\right)\right\}$, for all $i$ and $j$.

Case 7: The number of binary codes is put to the introducing of additional restriction of $\left(2^{n_{s}}-k_{s}\right)$ having in auxiliary and additional constraints in separately with different models. The auxiliary constraints are also depend open the range of coefficients in each sets of whose sum is nearer to the aspiration levels or choices. But the additional constraints in each models are expressed as the difference between the aspiration level and sum of any about sets of specified binomials coefficients.

$$
\begin{aligned}
z_{i j}^{1}+z_{i j}^{2}+\cdots+z_{i j}^{n_{s}} & \geq r_{s 1} \\
z_{i j}^{1}+z_{i j}^{2}+\cdots+z_{i j}^{n_{s}} & \leq r_{s 2}
\end{aligned}
$$

and

$$
z_{i j}^{j_{1}^{\prime}}+z_{i j}^{j_{2}^{\prime}}+\cdots+z_{i j}^{j_{s r 2}^{\prime}} \leq r_{s 2}-1
$$

The excess additional constraints should be imposed on $z_{i j}^{j_{1}^{\prime}}, z_{i j}^{j_{2}^{\prime}}, \cdots, z_{i j}^{j_{s r 2}^{\prime}} \in A_{i j}^{s r_{2} k}$ for all $i$ and $j$.

$$
\sum_{t=1}^{n_{s}} z_{i j}^{(t)} \geq 1, \quad t \in\left\{j_{1}^{\prime}, j_{2}^{\prime}, \cdots, j_{r s_{1}}^{\prime}\right\}
$$

Case 8: Formulate the equivalent mathematical model with additional restriction as:

$$
\begin{aligned}
& \min : z=\left[\sum_{i=1}^{{ }^{n_{s}} C_{r_{s 1}}} \sum_{j=1}^{{ }^{n_{s}} C_{r_{s 1}}} A_{i j}^{r_{s 1}} B_{i j}^{r_{s 1}} C_{i j}^{k}+\right. \\
& \sum_{i=1}^{n_{s}} \sum_{j=1}^{C_{r_{s 1}+1}} \sum_{i j}^{n_{s}} A_{r_{s 1}+1}^{r_{s 1}+1} B_{i j}^{r_{s 1}+1} C_{i j}^{\left\{{ }^{n_{s}} C_{r_{s 1}}+k\right\}}+\text {, } \\
& \cdots,+\sum_{i=1}^{{ }^{n_{s}}{ }_{r_{s 2}-1}} \sum_{j=1}^{{ }^{n_{s}} C_{r_{s 2}-1}} A_{i j}^{r_{s 2}-1} B_{i j}^{r_{s 2}-1} \\
& C_{i j}^{\left[\left\{{ }^{n_{s}} C_{r_{s 1}}+, \cdots,+{ }^{n_{s}} C_{r_{s 2}-2}\right\}+k\right]} \\
& \left.+\sum_{i=1}^{\left(k_{s}-L_{i j}^{1}\right)} \sum_{j=1}^{\left(k_{s}-L_{i j}^{1}\right)} A_{i j}^{r_{s 2}} B_{i j}^{r_{s 2}} C_{i j}^{\left(L_{i j}^{1}\right)+k}\right] x_{i j}, \\
& s=1,2, \cdots, K \\
& z_{i j}^{1}+z_{i j}^{2}+\cdots+z_{i j}^{n_{s}} \geq r_{s 1} \\
& z_{i j}^{1}+z_{i j}^{2}+\cdots+z_{i j}^{n_{s}} \leq r_{s 2} \\
& \text { and } z_{i j}^{j_{1}^{\prime}}+z_{i j}^{j_{2}^{\prime}}+\cdots+z_{i j}^{j_{s r 2}^{\prime}} \leq r_{s 2}-1
\end{aligned}
$$

where, $j^{\prime}=\left(k_{s}-L_{i j}^{(1)}\right)+1,\left(k_{s}-L_{i j}^{(1)}\right)+2$, $\ldots,{ }^{n_{s}} C_{r_{s 2}} x_{i j} \geq 0 ;$ for all $i$ and $j$. $z_{i j}^{n_{s}}=0 / 1, n_{s}=1,2, \cdots,\left\{\frac{\ln k_{s}}{\ln 2}\right\}$, and for all $i$ and $j$.

Where, $L_{i j}^{(1)}={ }^{n_{s}} C_{r_{s 1}}+{ }^{n_{s}} C_{r_{s 1}+1}+{ }^{n_{s}} C_{r_{s 1}+2}+$ $\cdots+{ }^{n_{s}} C_{r_{s 2}-1}$.

\subsection{Transformation technique: 2}

The proposed model is derived for restriction on the lower bound of binary variables. The Cases of $1,2,3,4$, and 5 are same as the above transformation of 1 .

Case 6: Assigned $k_{s}$ binary codes to $k_{s}$ number of aspiration levels for cost coefficients of the objective function.

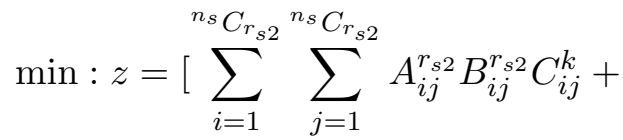

$$
\begin{aligned}
& \sum_{i=1}^{{ }_{s}} \sum_{j=1}^{n_{r_{s 2}-1}{ }^{n_{s}} C_{r_{s 2}-1}} A_{i j}^{r_{s 2}-1} B_{i j}^{r_{s 2}-1} C_{i j}^{\left\{{ }^{n_{s}} C_{r_{s 2}}+k\right\}}+\text {, }
\end{aligned}
$$

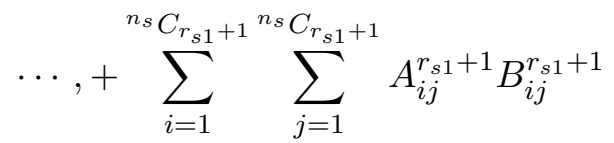

$$
\begin{aligned}
& C_{i j}^{\left.\left[{ }^{n_{s}} C_{r_{s 2}}+, \cdots,+{ }^{n_{s}} C_{r_{s 2}+2}\right\}+k\right]} \\
& \left.+\sum_{i=1}^{\left(k_{s}-L_{i j}^{2}\right)} \sum_{j=1}^{\left(k_{s}-L_{i j}^{2}\right)} A_{i j}^{r_{s 1}} B_{i j}^{r_{s 1}} C_{i j}^{\left(L_{i j}^{2}\right)+k}\right] x_{i j} \text {, } \\
& s=1,2, \cdots, K
\end{aligned}
$$


where, $L_{i j}^{(2)}={ }^{n_{s}} C_{r_{s 2}}+{ }^{n_{s}} C_{r_{s 2}-1}+{ }^{n_{s}} C_{r_{s 2}-2}+$ $\cdots+{ }^{n_{s}} C_{r_{s 2}+1}$, and $j_{1}^{\prime} \in\left\{1,2, \cdots,\left(n_{s}-s\right)+\right.$ $1\}, \quad j_{2}^{\prime} \in\left\{2,3, \cdots,\left(n_{s}-s\right)+2\right\}, \cdots, j_{s}^{\prime} \in$ $\left\{s, s+1, \cdots, n_{s}\right\}$

$I_{s}^{\left(j^{\prime}\right)}=\left\{\left(j_{1}^{\prime}, j_{2}^{\prime}, \cdots, j_{s}^{\prime}\right) / j_{1}^{\prime}<j_{2}^{\prime}<\cdots<j_{s}^{\prime} s=\right.$ $\left.r_{s 1}, r_{s 1+1}, r_{s 1+2}, \cdots, r_{s 2}\right\}$

where, $A_{i j}^{s_{k}}=\left\{z_{i j}^{\left(j_{1}^{\prime}\right)}, z_{i j}^{\left(j_{2}^{\prime}\right)}, \cdots, z_{i j}^{\left(j_{s}^{\prime}\right)} /\left(j_{1}^{\prime}, j_{2}^{\prime}, \cdots, j_{s}^{\prime}\right)\right.$ $\left.\in I_{s}^{\left(j^{\prime}\right)}\right\}$, where $s=r_{s 1}, r_{s 1}+1, r_{s 1}+2, \cdots, r_{s 2}$.

Therefore, $B_{i j}^{s_{k}}=\left\{\Pi_{k=1}^{n_{s}}\left(1-z_{i j}^{j_{k}}\right) / j_{k}^{\prime}\right.$ not belongs to $\left.\left(j_{1}^{\prime}, j_{2}^{\prime}, \cdots, j_{s}^{\prime}\right)\right\}$, for all $i$ and $j$.

Case 7: On restriction of $\left(2^{n_{s}}-k_{s}\right)$ with the number of binary codes in respect to the additional restriction having auxiliary and additional constraints as follows:

$$
\begin{gathered}
z_{i j}^{1}+z_{i j}^{2}+\cdots+z_{i j}^{n_{s}} \geq r_{s 1}, \\
\text { and } \quad z_{i j}^{1}+z_{i j}^{2}+\cdots+z_{i j}^{n_{s}} \leq r_{s 2},
\end{gathered}
$$

for all $i$ and $j$. The excess additional constraints should be imposed on $z_{i j}^{j_{1}^{\prime}}, z_{i j}^{j_{2}^{\prime}}, \cdots, z_{i j}^{j_{s 2}^{\prime}} \in$ $A_{i j}^{s r_{2} k}$ for all $i$ and $j$.

$\sum_{t=1}^{n_{s}} z_{i j}^{(t)} \geq 1, \quad t$ not belongs to $\left\{j_{1}^{\prime}, j_{2}^{\prime}, \cdots, j_{r s_{1}}^{\prime}\right\}$.

Case 8: Formulate the equivalent mathematical model with additional restriction as:

$$
\begin{aligned}
& \min : z=\left[\sum_{i=1}^{n_{s} C_{r_{s 2}}} \sum_{j=1}^{n_{s} C_{r_{s 2}}} A_{i j}^{r_{s 2}} B_{i j}^{r_{s 2}} C_{i j}^{k}+\right.
\end{aligned}
$$

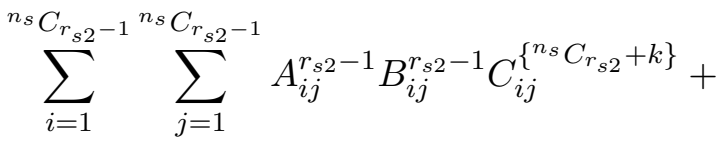

$$
\begin{aligned}
& \cdots+\sum_{i=1}^{n_{s}} \sum_{j=1}^{C_{r_{s 1}+1}} A_{i j}^{r_{s}+1} B_{i j}^{r_{s 1}+1} \\
& C_{i j}^{\left[\left\{{ }^{n_{s}} C_{r_{s 2}}+, \cdots,+{ }^{n_{s}} C_{r_{s 1}+2}\right\}+k\right]} \\
& \left.+\sum_{i=1}^{\left(k_{s}-L_{i j}^{(2)}\right)} \sum_{j=1}^{\left(k_{s}-L_{i j}^{(2)}\right)} A_{i j}^{r_{s 1}} B_{i j}^{r_{s 1}} C_{i j}^{\left(L_{i j}^{2}\right)+k}\right] x_{i j} \text {, } \\
& s=1,2, \cdots, K . \\
& z_{i j}^{1}+z_{i j}^{2}+\cdots+z_{i j}^{n_{s}} \geq r_{s 1}, \\
& z_{i j}^{1}+z_{i j}^{2}+\cdots+z_{i j}^{n_{s}} \leq r_{s 2},
\end{aligned}
$$

for all $i$ and $j$, where, $j^{\prime}=\left(k_{s}-L_{i j}^{(2)}\right)+1,\left(k_{s}-\right.$ $\left.L_{i j}^{(2)}\right)+2, \cdots,{ }^{n_{s}} C_{r_{s 1}} ; x_{i j} \geq 0 ; z_{i j}^{n_{s}}=0 / 1, n_{s}=$ $1,2, \cdots,\left\{\frac{\ln k_{s}}{\ln 2}\right\} ; L_{i j}^{(2)}={ }^{n_{s}} C_{r_{s 2}}+{ }^{n_{s}} C_{r_{s 2}-1}+$
${ }^{n_{s}} C_{r_{s 2}-2}+\cdots+{ }^{n_{s}} C_{r_{s 1}+1}$.

Remark: Multi-choice stochastic transportation problem can be transformed to an equivalent mathematical programming problem by using one of the above transformation technique, when the transformation technique is also established in any models, then the cases can be stated as:

Case-1: Different types of models also depend upon the different set of auxiliary equation which is raised by the set of conjugate coefficients in which sum is nearer to the aspiration levels as $k_{s}$ are ${ }^{n_{s}} C_{r_{s 1}},{ }^{n_{s}} C_{r_{s 1}+1},{ }^{n_{s}} C_{r_{s 1}+2}, \cdots,{ }^{n_{s}} C_{n_{s}}$.

Case-2: The additional constraints lead to the sum of conjugate terms (which is just nearer to the choices) - $k_{s}$ (aspiration levels) greater than 0 .

Case-3: In case of $k_{s}=2^{n_{s}}$ involving binary variables, then the expression will be $2^{n_{s}}={ }^{n_{s}} C_{0}+{ }^{n_{s}} C_{1}+{ }^{n_{s}} C_{2}+\cdots+{ }^{n_{s}} C_{n_{s}}$ restriction will be as

$$
z_{i j}^{1}+z_{i j}^{2}+\cdots+z_{i j}^{n_{s}} \neq 0
$$

and

$$
z_{i j}^{1}+z_{i j}^{2}+\cdots+z_{i j}^{n_{s}} \neq n_{s},
$$

for all $i$ and $j$, hence the above two restriction will not effect on the solution procedure of the mathematical model.

Case-4: When $k_{s} \neq 2^{n_{s}}$, then from the expression of $2^{n_{s}}$ the smallest number of conjugative terms whose sum is equal or just greater than $k_{s}$ are ${ }^{n_{s}} C_{r_{s 1}},{ }^{n_{s}} C_{r_{s 1}+1},{ }^{n_{s}} C_{r_{s 1}+2}, \cdots,{ }^{n_{s}} C_{n_{s}}$, Hence $k_{s} \leq{ }^{n_{s}} C_{r_{s 1}},{ }^{n_{s}} C_{r_{s 1}+1},{ }^{n_{s}} C_{r_{s 1}+2}, \cdots,{ }^{n_{s}} C_{n_{s}}, s=$ $1,2, \cdots, k$. In this case four possibilities may be arises.

\section{Case Study}

A reputed cold drinks supply company transports the cold drinks from three product center at Dankuni, Howrah, Asanshol to the four destination center at Jhargram, Kharagpur, Trakeshar and Contai. In summer season, the cold drinks has tremendous demand every time at four destination centers. The transportation time cost is an important factor of transportation planning programme. The transportation time cost is related with fluctuation of competency of driver, road condition etc. The manufacturing time at production centers depends upon the availability of current supply and machine condition and skilled mechanical hands etc. Delivery time is 
related with transporting system of smooth distribution of product in due time at destination center. Due to fluctuation of above factor, transportation cost time on each route is not fixed. They are appended below in Table- 1 :

Table 1. Multi-choice transportation time cost (in Hours) $C_{i j}^{k}$ : per unit (1 unit $=$ Milage distance) for route $x_{i j}$

\begin{tabular}{llr}
\hline Sl. No. & Route: $x_{i j}$ & Transportation time cost \\
\hline 1 & $(1,1): x_{11}$ & 12 or 13 or 14 \\
2 & $(1,2): x_{12}$ & 15 or 16 \\
3 & $(1,3): x_{13}$ & 19 or 20 or 21 or 22 \\
4 & $(1,4): x_{14}$ & 24 or 25 or 26 \\
5 & $(2,1): x_{21}$ & 16 or 18 or $\cdots$ or 24 or 26 \\
6 & $(2,2): x_{22}$ & 17 or 18 or $\cdots$ or 21 or 22 \\
7 & $(2,3): x_{23}$ & 9 or 10 or 11 \\
8 & $(2,4): x_{24}$ & 17 or 18 \\
9 & $(3,1): x_{31}$ & 18 or 19 or $\cdots$ or 23 or 24 \\
10 & $(3,2): x_{32}$ & 12 or 13 or $\cdots$ or 18 or 19 \\
11 & $(3,3): x_{33}$ & 25 or 26 or 27 \\
12 & $(3,4): x_{34}$ & 28 or 29 \\
\hline
\end{tabular}

A multi-choice stochastic transportation problem is presented where the objective function and the constraints are formulated as:

$$
\begin{array}{r}
\min : z=\sum_{i=1}^{3} \sum_{j=1}^{4}\left\{C_{i j}^{1}, C_{i j}^{2}, \cdots, C_{i j}^{k}\right\} x_{i j}, \\
k=1,2, \cdots, 10 \\
\text { subject to } \quad \operatorname{Pr}\left(\sum_{j=1}^{4} x_{i j} \leq s_{i}\right) \geq 1-\gamma_{i}, \\
i=1,2,3 \\
\operatorname{Pr}\left(\sum_{i=1}^{3} x_{i j} \geq d_{j}\right) \geq 1-\delta_{j}, \\
j=1,2,3,4
\end{array}
$$

where, $x_{i j} \geq 0, i=1,2,3 ; j=1,2,3,4$ and

$$
0<\gamma_{i}<1,0<\delta_{j}<1
$$

In the case study, three known parameters $s_{1}, s_{2}, s_{3}$ of Weibull distribution is consider. The specified probability levels with shape and scale parameters of supplies $s_{1}, s_{2}, s_{3}$ are given in the Table -2 .
Table 2. Value of shape and scale parameters with Specified prob.levels (SPL) of $s_{i}$.

\begin{tabular}{llr}
\hline Shape parameters & Scale parameters & (SPL) \\
\hline$\alpha_{1}=3000$ & $\beta_{1}=3.6$ & $\gamma_{1}=0.01$ \\
$\alpha_{2}=2500$ & $\beta_{2}=3.0$ & $\gamma_{2}=0.02$ \\
$\alpha_{3}=2000$ & $\beta_{3}=2.4$ & $\gamma_{3}=0.03$ \\
\hline
\end{tabular}

Further, the specified probability levels and the shape and the scale parameters of demands parameters $d_{1}, d_{2}, d_{3}, d_{4}$ which follow Weibull distribution are given in Table -3 .

Table 3. Value of shape and scale parameters with Specified prob.levels (SPL) of $d_{j}$.

\begin{tabular}{llr}
\hline Shape parameters. & Scale parameters & $(\mathrm{SPL})$ \\
\hline$\alpha_{1}^{\prime}=1700$ & $\beta_{1}^{\prime}=2.2$ & $\delta_{1}=0.04$ \\
$\alpha_{2}^{\prime}=1500$ & $\beta_{2}^{\prime}=2.0$ & $\delta_{2}=0.05$ \\
$\alpha_{3}^{\prime}=1250$ & $\beta_{3}^{\prime}=1.6$ & $\delta_{3}=0.06$ \\
$\alpha_{4}^{\prime}=1000$ & $\beta_{4}^{\prime}=1.2$ & $\delta_{4}=0.07$ \\
\hline
\end{tabular}

Using the data provided in Table 1,2 and 3 the following deterministic multi-choice transportation problem is formulated as:

$$
\begin{array}{r}
\min : z=\{12,13,14\} x_{11}+\{15,16\} x_{12} \\
+\{19,20,21,22\} x_{13}+\{24,25,26\} x_{14} \\
+\{16,18,20,22,24,26\} x_{21} \\
+\{17,18,19,20,21,22\} x_{22} \\
+\{9,10,11\} x_{23}+\{17,18\} x_{24} \\
+\{18,19,20,21,22,23,24\} x_{31} \\
+\{12,13,14,15,16,17,18,19\} x_{32} \\
+\{25,26,27\} x_{33}+\{28,29\} x_{34} \\
\text { subject to } \quad \sum_{j=1}^{4} x_{1 j} \leq 3829.430743 \\
\sum_{j=1}^{4} x_{2 j} \leq 3404.6222029 \\
\sum_{j=1}^{4} x_{3 j} \leq 3346.480624 \\
\sum_{i=1}^{3} x_{i 1} \geq 2892.168344 \\
\sum_{i=1}^{3} x_{i 2} \geq 2860.129000
\end{array}
$$




$$
\begin{aligned}
& \sum_{i=1}^{3} x_{i 3} \geq 2386.056503 \\
& \sum_{i=1}^{3} x_{i 4} \geq 2259.265858
\end{aligned}
$$

$$
x_{i j} \geq 0, \quad i=1,2,3 \text { and } j=1,2,3,4 .
$$

Now using a new transformation technique, the following a multi-choice deterministic transportation problem is obtained as bellow:

$$
\begin{aligned}
\min : z= & t_{11} x_{11}+t_{12} x_{12}+t_{13} x_{13}+t_{14} x_{14} \\
& +t_{21} x_{21}+t_{22} x_{22}+t_{23} x_{23}+t_{24} x_{24} \\
& +t_{31} x_{31}+t_{32} x_{32}+t_{33} x_{33}+t_{34} x_{34}
\end{aligned}
$$

subject to $(48)-(54)$

where

$$
\begin{aligned}
& t_{11}=12 z_{11}^{1} z_{11}^{2} \\
& +13 z_{11}^{1}\left(1-z_{11}^{2}\right)+14\left(1-z_{11}^{1}\right) z_{11}^{2} \\
& t_{12}=15 z_{12}^{1}+16\left(1-z_{12}^{1}\right) \\
& t_{13}=19 z_{13}^{1} z_{13}^{2}+20 z_{13}^{1}\left(1-z_{13}^{2}\right) \\
& +21\left(1-z_{13}^{1}\right) z_{13}^{2}+22\left(1-z_{13}^{1}\right)\left(1-z_{13}^{2}\right) \\
& t_{14}=25 z_{14}^{1} z_{14}^{2}+26 z_{14}^{1}\left(1-z_{14}^{2}\right) \\
& +27\left(1-z_{14}^{1}\right) z_{14}^{2} \\
& t_{21}=16 z_{21}^{1}\left(1-z_{21}^{2}\right)\left(1-z_{21}^{3}\right) \\
& +18\left(1-z_{21}^{1}\right) z_{21}^{2}\left(1-z_{21}^{3}\right) \\
& +20\left(1-z_{21}^{1}\right)\left(1-z_{21}^{2}\right) z_{21}^{3} \\
& +22 z_{21}^{1} z_{21}^{2}\left(1-z_{21}^{3}\right) \\
& +24\left(1-z_{21}^{1}\right) z_{21}^{2} z_{21}^{3}+26 z_{21}^{1}\left(1-z_{21}^{2}\right) z_{21}^{3} \\
& t_{22}=17 z_{22}^{1}\left(1-z_{22}^{2}\right)\left(1-z_{22}^{3}\right) \\
& +18\left(1-z_{22}^{1}\right) z_{22}^{2}\left(1-z_{22}^{3}\right)+19 z_{22}^{1} z_{22}^{2}\left(1-z_{22}^{3}\right) \\
& +20\left(1-z_{22}^{1}\right)\left(1-z_{22}^{2}\right) z_{22}^{3}+21 z_{22}^{1}\left(1-z_{22}^{2}\right) z_{22}^{3} \\
& +22\left(1-z_{22}^{1}\right) z_{22}^{2} z_{22}^{3} \\
& t_{23}=9 z_{23}^{1} z_{23}^{2}+10 z_{23}^{1}\left(1-z_{23}^{2}\right) \\
& +11\left(1-z_{23}^{1}\right) z_{23}^{2} \\
& t_{24}=17 z_{24}^{1}+18\left(1-z_{24}^{2}\right) \\
& t_{31}=18\left(1-z_{31}^{1}\right)\left(1-z_{31}^{2}\right)\left(1-z_{31}^{3}\right) \\
& +19 z_{31}^{1}\left(1-z_{31}^{2}\right)\left(1-z_{31}^{3}\right) \\
& +20\left(1-z_{31}^{1}\right) z_{31}^{2}\left(1-z_{31}^{3}\right) \\
& +21\left(1-z_{31}^{1}\right)\left(1-z_{31}^{2}\right) z_{31}^{3} \\
& +22 z_{31}^{1} z_{31}^{2}\left(1-z_{31}^{3}\right)+23 z_{31}^{1}\left(1-z_{31}^{2}\right) z_{31}^{3} \\
& +24\left(1-z_{31}^{1}\right) z_{31}^{2} z_{31}^{3} \\
& t_{32}=12 z_{32}^{1} z_{32}^{2} z_{32}^{3}+13\left(1-z_{32}^{1}\right) z_{32}^{2} z_{32}^{3} \\
& +14 z_{32}^{1}\left(1-z_{32}^{2}\right) z_{32}^{3} \\
& +15 z_{32}^{1} z_{32}^{2}\left(1-z_{32}^{3}\right)+16\left(1-z_{32}^{1}\right)\left(1-z_{32}^{2}\right) z_{32}^{3} \\
& +17 z_{32}^{1}\left(1-z_{32}^{2}\right)\left(1-z_{32}^{3}\right) \\
& +18\left(1-z_{32}^{1}\right) z_{32}^{2}\left(1-z_{32}^{3}\right)
\end{aligned}
$$

\section{Conclusion}

This paper proposes a solution procedure to multi-choice stochastic unbalanced transportation problem involving multiplicative terms of binary variables in all cost coefficient of the objective function and subject to some probabilistic

where

$$
x_{i j} \geq 0, \quad i=1,2,3 \text { and } j=1,2,3,4 .
$$

The above mathematical programming model is treated as a non-linear mixed integer programming problem, which is solved by using Lingo 11.0 [16] software. The optimal solution is obtained as : $\quad x_{11}=2892.168, x_{14}=937.2624, x_{23}=$ $2386.057, x_{24}=1018.566, \quad x_{32}=2860.139, x_{34}=$ 303.4378 where rest of the decision variables are zero. The minimum time cost of the objective function is 138808.44. For the optimal value of the objective function, the multi-choice cost coefficients are obtained as follows:

\begin{tabular}{lllcccc}
\hline$x_{i j}$ & $x_{11}$ & $x_{12}$ & $x_{13}$ & $x_{14}$ & $x_{21}$ & $x_{22}$ \\
& $x_{23}$ & $x_{24}$ & $x_{31}$ & $x_{32}$ & $x_{33}$ & $x_{34}$ \\
\hline value of $C_{i j}^{k}$ & 12 & 15 & 19 & 24 & 16 & 19 \\
& 9 & 17 & 24 & 12 & 27 & 28 \\
\hline
\end{tabular}

If the decision variables $x_{i j}, i=1,2,3 ; j=$ $1,2,3,4$ are considered to be integers, the optimal solution can be obtained as : $x_{11}=2893, x_{14}=$ $936, x_{23}=2387, x_{24}=1017, \quad x_{32}=2861, x_{34}=$ 307 where rest of the decision variables are zero. The minimum time cost of the objective function is 138880.00 . For the optimal value of the objective function, the multi-choice cost coefficients are obtained as follows:

\begin{tabular}{llccccc}
\hline$x_{i j}$ & $x_{11}$ & $x_{12}$ & $x_{13}$ & $x_{14}$ & $x_{21}$ & $x_{22}$ \\
& $x_{23}$ & $x_{24}$ & $x_{31}$ & $x_{32}$ & $x_{33}$ & $x_{34}$ \\
\hline value of $C_{i j}^{k}$ & 12 & 15 & 19 & 24 & 16 & 18 \\
& 9 & 17 & 24 & 12 & 25 & 28 \\
& & & & & &
\end{tabular}


linear constraints associated with Weibull distribution. The binary variables for each choices are dependent on relation $\frac{\ln k_{i}}{\ln (2)}$, where $k_{i}$ is the number of choices.

Initially, all the stochastic constraints are transformed into equivalent deterministic constraints. In this paper, the generalized multi-choice programming technique has been incorporated, where the multi-choice type cost coefficients are handled by transferring into an equivalent deterministic model. The additional restrictions involving binary variables are used in some cases of the transformed models to restrict the repetition of goals. After introduction the auxiliary and additional constraints in terms of binary variables with multi-choice parameters are transformed into mixed integer programming problem. In this paper, the three models such as $\mathbf{2}$ and $\mathbf{3}$ and $\mathbf{4}$ are also established, and would be obtained the optimal solution in each models. But now the last case (Model-4) is illustrated with a numerical example. A further study is needed for the models with multi-choice parameters in coefficients of variables in source and destination constraints, and cost coefficients also follow the randomness depending on the problems.

\section{Acknowledgments}

The author is very grateful to the referee's for their valuable comments to revise the paper

\section{References}

[1] Acharya, S and Acharya, M.M., Generalized transformation technique for multi-choice linear programming problem. An International Journal of Optimization and Control: Theories \& Applications, 3(1), 45-54 (2013).

[2] Biswal, M.P. and Acharya, S., Transformation of a multi-choice linear programming problem. Applied Mathematics and Computation, 210, 182-188 (2009).

[3] Biswal, M.P. and Acharya, S., Solving probabilistic programming problems involving multi-choice parameters. Opsearch, 210, 1-19 (2011).

[4] Chang, C.-T., Multi-choice goal programming. Omega, The International Journal of Management Science., 35, 389-396 (2007).

[5] Chang, C.-T., Binary fuzzy programming. European Journal of Operation Research, 180, 29-37 (2007).

[6] Chang, C.-T., Revised multi-choice goal programming. Applied Mathematical Modeling, 32, 2587-2595 (2008).
[7] Chang, Ching-Ter, Chen, Huang-Mu , and Zhuang, Zheng-Yun, Multi-coefficients goal programming. Computers and Industrial Engineering, 62(2), 616-623 (2012).

[8] Liao, Chin-Nung, Revised multi-segment goal programming and applications. Prob. Stat. Forum, 4, 110-119 (2011).

[9] Fok, S.L. and Smart, J., Accuracy of failure predictions based on weibull statistic. J. Eur. Ceram. Soc., 15, 905-908 (1995).

[10] Goicoechea, A., Hansen, D.R. and Duckstein, L., Multi-objective Decision Analysis with Engineering and Business Application. New York: John Wiley and Sons (1982).

[11] Hitchcock, F.L., The distribution of a product from several sources to numerous localities. J. Math. Physics, 20, 224-230 (1941).

[12] Koopmans, T.C., Optimum utilization of the transportation system. Ecpnometrica, 17, 3-4 (1949).

[13] Mahapatra, D.R., Roy, S.K. and Biswal, M.P., Computation of multi-objective stochastic transportation problem involving normal distribution with joint constraints. The Journal of Fuzzy Mathematics. 19(4), 865-876 (2011).

[14] Mahapatra, D.R., Roy, S.K. and Biswal, M.P., Multi-choice stochastic transportation problem involving extreme value distribution. Applied Mathematical Modelling, 37(4), 2230-2240 (2013).

[15] Rabindran, A., Philips, D.T. and Solberg, J.J., Operations Research; Principles and Practice. Second Edition, New York: John Wiley and Sons (1987).

[16] Schrage, L., Optimization Modeling with LINGO. LINDO System, Chicago, I11, USA, 6 th edition.

[17] Weibull, W., A statistical distribution function of wide applicability. J. of Appl. Mach., 15, 293-302 (1951).

Deshabrata Roy Mahapatra was born in India. He received B.Sc (Hons) in mathematics; Bachelor of Education and M.Sc (Applied Mathematics) degrees from Vidyasagar University and Kalyani University respectively. He devoted himself during 1996 - 1998 academic year as a guest lecturer in Mathematics Department of Palpara College. He was selected as a teacher of mathematics two times in 1998 and 1999 on basis of State Level Selection test in West Bengal and since then he has been working till now. In 2001, he had been successfully qualified in eligible test 
for Research Fellowship and Lectureship in $\mathrm{Na}$ tional and State level. He has published over 11 refereed journal and International conference papers in the area of Transportation planning, Stochastic programming, Fuzzy programming, Interval order relation and Multi-choice programming. His research interests include Game theory and Numerical analysis. 